\title{
Synergistic protection of bone vasculature and bone mass by desferrioxamine in osteoporotic mice
}

\author{
LIANG WANG $^{1,2^{*}}$, PENG JIA $^{1 *}$, YU SHAN $^{3 *}$, YANMING HAO $^{4 *}$, XIAO WANG $^{1}$, YU JIANG $^{1}$, YE YUAN $^{1}$, \\ QIAOQIAO DU ${ }^{1}$, HUI ZHANG ${ }^{1}$, FAN YANG $^{1}$, WEN ZHANG $^{5}$, MAO SHENG $^{2}$ and YOUJIA XU ${ }^{1}$ \\ ${ }^{1}$ Department of Orthopaedics, The Second Affiliated Hospital of Soochow University, Suzhou, Jiangsu 215004; \\ ${ }^{2}$ Department of Radiology, Children's Hospital of Soochow University, Suzhou, Jiangsu 215123; \\ ${ }^{3}$ Department of Orthopaedics, The First People's Hospital of Wujiang, Suzhou, Jiangsu 215200; ${ }^{4}$ Department of \\ Orthopaedics, The First People's Hospital of Kunshan, Suzhou, Jiangsu 215300; ${ }^{5}$ Orthopaedic Institute of \\ Soochow University, Suzhou, Jiangsu 215006, P.R. China
}

Received March 26, 2017; Accepted August 16, 2017

DOI: $10.3892 / \mathrm{mmr} .2017 .7451$

\begin{abstract}
It has previously been demonstrated that impaired angiogenesis is associated with metabolic abnormalities in bone in addition to osteoporosis (including postmenopausal osteoporosis). Enhancing vessel formation in bone is therefore a potential clinical therapy for osteoporosis. The present study conducted an in-depth investigation using desferrioxamine (DFO) in an ovariectomy (OVX)-induced osteoporotic mouse model in order to determine the time frame of alteration of bone characteristics and the therapeutic effect of DFO. It was demonstrated that OVX induced instant bone mass loss 1 week following surgery, as expected. In contrast, DFO treatment protected the mice against OVX-induced osteoporosis during the first week, however failed to achieve long-term protection at a later stage. A parallel alteration for cluster of differentiation 31/endomucin double positive vessels (type $\mathrm{H}$ vessels) was observed, which have previously been reported to be associated with osteogenesis. DFO administration not only partially prevented bone loss and maintained trabecular bone microarchitecture, however additionally enhanced the type $\mathrm{H}$ vessels during the first week post-OVX. The molecular mechanism of how DFO influences type $\mathrm{H}$ vessels to regulate bone metabolism needs to be further investigated. However,
\end{abstract}

Correspondence: Dr Mao Sheng, Department of Radiology, Children's Hospital of Soochow University, 92 Zhongnan Street, Suzhou Industrial Park, Suzhou, Jiangsu 215123, P.R. China

E-mail: shengmaosz@163.com

Dr Youjia Xu, Department of Orthopaedics, The Second Affiliated Hospital of Soochow University, 1055 Sanxiang Road, Suzhou, Jiangsu 215004, P.R. China

E-mail: xuyoujia@medmail.com.cn

*Contributed equally

Key words: mouse model, osteoporosis, desferrioxamine, angiogenesis, bone mass the findings of the present study provide preliminary evidence to support combined vascular and osseous therapies for osteoporotic patients. Pharmacotherapy may offer a novel target for improving osteoporosis by promoting type $\mathrm{H}$ vessel formation, which indicates potential clinical significance in the field of bone metabolism.

\section{Introduction}

Osteoporosis, which usually leads to bone fractures due to fragility, is a major public health problem, especially in the elderly population. Osteoporosis is generally characterized by low bone mass and microarchitectural deterioration of bone tissue (1) as a result of metabolic bone disorders. Studies focusing on the etiology, prevention, and treatment of osteoporosis have been widely conducted, and significant progress has been made during the last two decades (2-8). A number of key effectors such as NF- $\kappa$ B, RANKL, Wnt5a/Ror2, and PPAR $\beta / \delta$ have been identified to be pivotal in the regulation of bone metabolism (9-12).

On the other hand, bone is a highly vascularized tissue, and blood vessels participate in bone development, remodeling, and homeostasis (13). Bone vasculature not only supplies bone tissue with oxygen, nutrients, and growth factors but also removes waste products and delivers osteoprogenitors to fracture sites $(14,15)$. Activation of the hypoxia-inducible factor (HIF) pathway can accelerate bone regeneration and improve bone healing in vitro and in vivo (16). This suggests intercellular signaling between osteoprogenitors and endothelial precursor cells, which has been also termed as a coupling of osteogenesis and angiogenesis $(17,18)$. Recently, a distinct capillary subtype termed the type $H$ vessel was found in murine bone metaphysis and periosteum, which couples osteogenesis and angiogenesis. Type $\mathrm{H}$ vessels, strongly positive for endothelial cell surface markers, CD31 and Endomucin $\left(\mathrm{CD} 31^{\mathrm{hi}} \mathrm{Emcn}^{\mathrm{hi}}\right)$, can mediate local bone vascular growth and also maintain perivascular osteoprogenitors (19).

Desferrioxamine (DFO) can promote the differentiation of mesenchymal stem cells (MSCs) by activating a $\beta$-catenin signaling pathway (20). DFO also enhances normal 
fracture healing when injected locally into a fracture callus by increasing endothelial tubule formation (21). Recent reports also indicated that DFO administration can prevent age-dependent loss of type $\mathrm{H}$ vessels thus to improve the bone quality in aged mice (19). Given all these facts, we propose that DFO treatment theoretically have potential to activate angiogenesis thus to help maintain bone mass, which would be especially beneficial to prevent or treat osteoporosis. To verify the hypothesis, ovariectomized (OVX) mice were used as animal model here in this study to investigate the effect of DFO on OVX-induced osteoporosis.

\section{Materials and methods}

Mice. The C57BL/6 mice used in this study were provided by National Resource Center of Model Mice of Nanjing University and housed in the specific pathogen free barrier system in the animal facility of Soochow University. All animal-related study was performed in compliance with the relevant laws and internal guidelines of the Ethics Committee of the Second Affiliated Hospital of Soochow University (approval no. sdfey160215). All surgery was performed under avertin anesthesia and all efforts were made to minimize suffering.

Osteoporotic mouse model. Eight-week-old female mice were randomly divided into 3 groups. All mice were anesthetized and subjected to bilateral OVX or a sham operation. After a 1-week recovery period, the sham group mice were administrated intraperitoneal saline (sham group) while the OVX mice were administered saline (OVX group) or DFO (OVX + DFO group) (Sigma-Aldrich; Merck KGaA, Darmstadt, Germany). For DFO treatment, freshly prepared DFO in $0.9 \%$ saline $[15 \mathrm{mg} / \mathrm{ml}$ per mouse as previous reported (19)] was administrated every other day for 4 weeks. Mouse tissues were collected at week 1, 2, 3 and 4 for investigation of vascular and osseous changes by immunostaining, hematoxylin and eosin staining, and microcomputed tomography (micro-CT) scanning.

Micro-CT analysis. Mouse femora and lumbar vertebrae were dissected at several points in time, and any attached soft tissue was completely removed. Collected bone specimens were then fixed in $4 \%$ paraformaldehyde (PFA) and analyzed by micro-CT (SkyScan 1176; Kontich, Belgium). The scanners were set at a voltage of $50 \mathrm{kV}$, a current of $500 \mu \mathrm{A}$, and a resolution of $9 \mu \mathrm{m}$. Image software (NRecon v1.6) and data analysis software (CTAn v1.13.11.0) were used for three-dimensional (3D) reconstruction of the trabecular bone.

After scanning, regions of interest (ROI) were defined by analysis software and limited to the trabecular regions. 3D trabecular bone images were reconstructed based on the ROI. Mouse femoral ROI were drawn starting from $540 \mu \mathrm{m}$ proximally to the distal growth plate over $1.35 \mathrm{~mm}$ toward the diaphysis. Lumbar vertebra ROI was drawn in the middle trabecular area with $2 \mathrm{~mm}$ of thickness. Trabecular bone parameters were calculated, including BMD $\left(\mathrm{g} / \mathrm{cm}^{3}\right)$, BV/TV (\%), Tb.Th (mm), Tb.N (per mm) and Tb.Sp (mm).

Bone tissue processing and immunostaining. For staining of bone sections, freshly collected specimens were fixed in $4 \%$
PFA overnight at $4^{\circ} \mathrm{C}$. Ethylenediaminetetraacetic acid solution was then applied for decalcification for at least 4 weeks. Tissues were then dehydrated using $20 \%$ sucrose and $2 \%$ polyvinylpyrrolidone solution for 2 weeks at $4^{\circ} \mathrm{C}$ and embedded in O.C.T. compound (Tissue-Tek). Cryosections $(10 \mu \mathrm{m})$ were prepared using a freezing microtome (Leica, Wetzlar, Germany) for immunostaining.

For immunostaining, bone sections were washed 3 times with $0.3 \%$ phosphate-buffered saline with Triton X-100 (PBST) and blocked with $5 \%$ bovine serum albumin in PBST. Sections were incubated with primary antibodies at $4{ }^{\circ} \mathrm{C}$ overnight. After washing 3 times with $0.3 \%$ PBST, sections were then incubated with fluorescein conjugated secondary antibodies together with nuclear counterstaining dye (DAPI) at room temperature. After another 3 thorough washes, slides were sealed with $50 \%$ glycerol and nail polish.

Antibodies. Primary antibodies used include rabbit-anti-mouse osterix (sc-22536-R) and rat-anti-mouse endomucin (sc-65495) (both from Santa Cruz Biotechnology, Inc., Dallas, TX, USA), and Alexa Fluor 488-conjugated anti-mouse CD31 (FAB3628 G; R\&D Systems, Inc., Minneapolis, MN, USA). Cy3- or Alexa 647-conjugated secondary antibodies (Molecular Probes, Eugene, OR, USA) were used for visualization.

Statistical analysis. All data are expressed as mean \pm SD. Statistical analyses were carried out using one-way analysis of variance with Student-Newman-Keuls post hoc test for multiple comparisons. $\mathrm{P}<0.05$ was considered to indicate a statistically significant difference $(\mathrm{P}<0.01 ; \mathrm{P}<0.0001)$. All statistical analyses were performed using SAS 8.2 software (SAS Institute Inc., Cary, NC, USA).

\section{Results}

Partial recovery of ovariectomy-induced osteoporosis in mouse femora by DFO administration. OVX female mice are frequently used as animal models for osteoporosis studies. In this study, we also used OVX female mice to investigate the detailed etiology and the therapeutic effect of DFO on osteoporosis. All mice were anesthetized and subjected to bilateral OVX or a sham operation (sham). After recovery, the Sham group mice were administrated with saline intraperitoneally (sham group), while the OVX mice were administered with saline (OVX group) or deferoxamine mesylate (OVX + DFO group; $15 \mathrm{mg} / \mathrm{ml}$ per mouse) every other day for 4 weeks. Mice were sacrificed at week 1, 2, 3 and 4 for $\mu \mathrm{CT}$ and histological evaluation. Based on $\mu \mathrm{CT}$ and quantification results, OVX induced remarkable reduction of bone mineral density (BMD) in mice femora, beginning at the first week. As shown in the reconstructed $\mu \mathrm{CT}$ images, the microarchitecture of the femora in OVX mice at week 1 was already significantly impaired (Fig. 1A and B). On the other hand, mice treated with deferoxamine (OVX + DFO group) showed slower reduction of BMD. The OVX + DFO group mice maintained comparable femoral BMD with the sham group at week 1, which began to decrease at week 2 and reached a similar BMD to the OVX group at week 3 (Fig. 1C). Quantification of BMD and other parameters also showed consistent results (Fig. 1D-H; Table I). In 

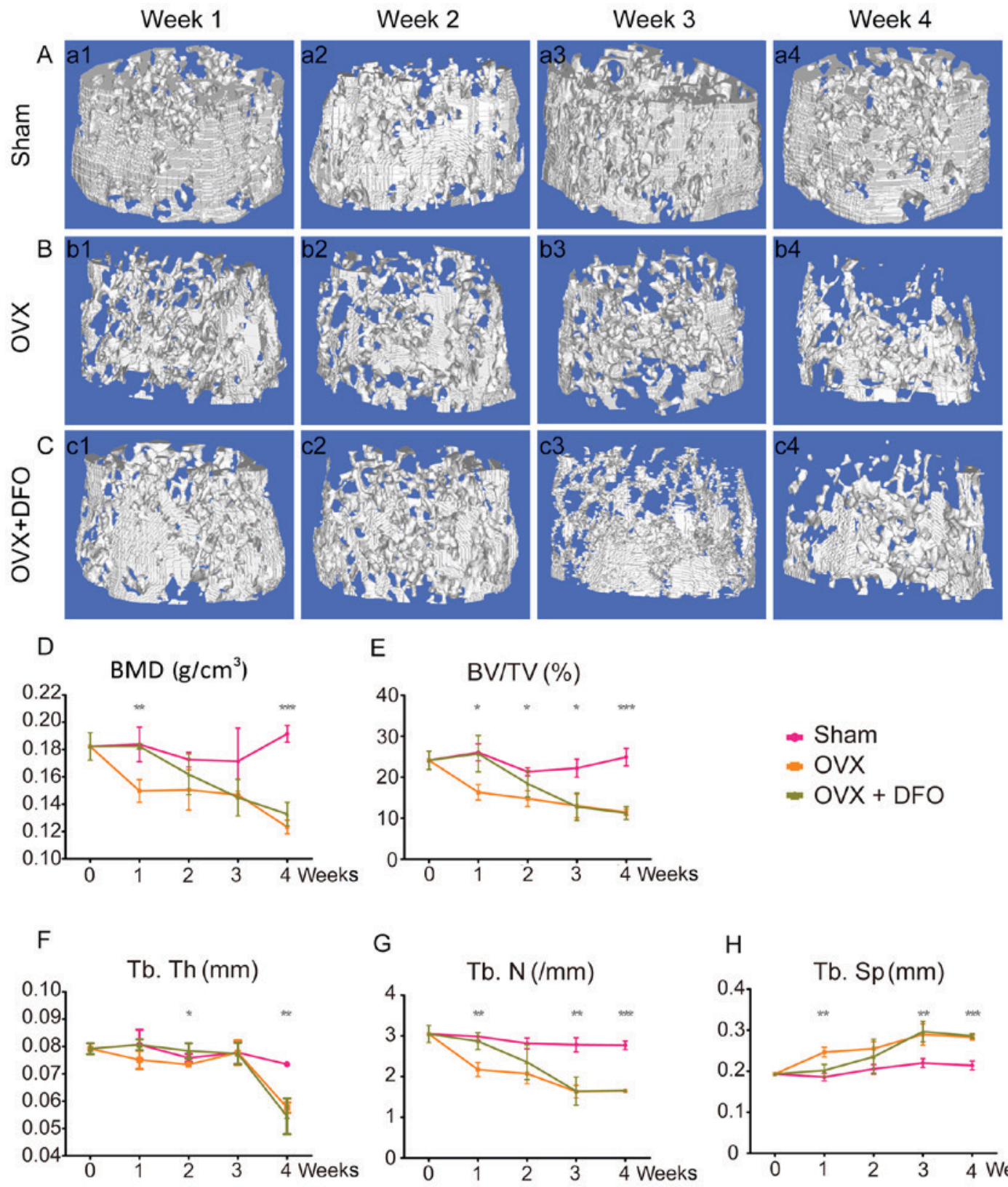

G
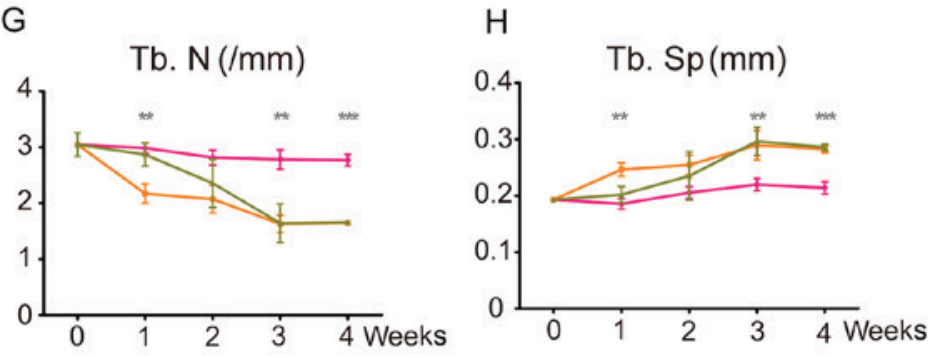

Figure 1. Micro-CT and quantification of bone microarchitecture in distal femur. (A-C) 3D micro-CT reconstructed images of the distal femora from sham group (A-a1-a4), OVX group (B-b1-b4) and OVX + DFO group (C-c1-c4) at different time-points (week 1, 2, 3 and 4). (D-H) Quantification of BMD (D), BV/TV (E), Tb.Th (F), Tb.N (G) and Tb.Sp (H) from the sham, OVX and OVX + DFO mice. " $\mathrm{P}<0.05 ;{ }^{* *} \mathrm{P}<0.01$; ${ }^{* * * *} \mathrm{P}<0.0001$. Micro-CT, micro-computed tomography; OVX, ovariectomy; DFO, desferrioxamine; BMD, bone mineral density; BV/TV, bone volume/tissue volume; Tb.Th, trabecular thickness; Tb.N, trabecular number; Tb.Sp, trabecular separation.

addition, the histomorphological changes of the distal femora were evaluated by hematoxylin and eosin staining. OVX mice showed less trabecular density at the distal ends of the femora compared to the sham group, and DFO treatment attenuated this effect at week 1 , which showed similar results comparable with $\mu \mathrm{CT}$ scanning (Fig. 2). The change of bone density in the OVX + DFO group indicated a temporary protective effect of DFO against OVX-induced osteoporosis.

Differential effect of DFO in the vertebrae of OVX mice. As indicated by a slight decline in BMD, OVX also induced osteoporosis in mouse lumbar vertebrae similar to that in the femur (Fig. 3A and B). The BMD and trabecular microarchitecture of lumbar vertebrae remarkably deteriorated in OVX mice compared to the sham group, which also began from week 1 but significantly changed at week 3 . However, DFO failed to protect the bone mass loss induced by OVX (Fig. 3C). The lumbar vertebrae BMD and microarchitecture of the OVX + DFO group mice also began to decrease at week 1 , with a similar trend to the OVX group mice (Fig. 3D-H; Table II). The BMD and microarchitecture results of the OVX + DFO group are similar to that of the OVX group, suggesting that DFO at this dose had no effect at weeks 3-4.

Vascular change correlated with osseous changes in the osteoporotic model. As reported, blood vasculature is essential during bone mass maintenance and fracture repair (13-15). A recent study characterized a distinct subtype 
Table I. BMD and trabecular microarchitecture of femur at different time-points.

\begin{tabular}{|c|c|c|c|c|c|c|}
\hline Parameters & Group & Week 0 & Week 1 & Week 2 & Week 3 & Week 4 \\
\hline \multirow[t]{3}{*}{$\operatorname{BMD}\left(\mathrm{g} / \mathrm{cm}^{3}\right)$} & Sham & $0.182 \pm 0.010$ & $0.184 \pm 0.013^{b}$ & $0.173 \pm 0.005$ & $0.171 \pm 0.024$ & $0.192 \pm 0.006^{\mathrm{b}}$ \\
\hline & OVX & $0.182 \pm 0.010$ & $0.149 \pm 0.008^{\mathrm{a}}$ & $0.150 \pm 0.014$ & $0.147 \pm 0.002$ & $0.123 \pm 0.005^{\mathrm{a}}$ \\
\hline & $\mathrm{OVX}+\mathrm{DFO}$ & $0.182 \pm 0.010$ & $0.182 \pm 0.002^{\mathrm{b}}$ & $0.162 \pm 0.015$ & $0.145 \pm 0.013$ & $0.133 \pm 0.008^{a}$ \\
\hline \multirow[t]{3}{*}{ BV/TV (\%) } & Sham & $24.135 \pm 2.221$ & $26.054 \pm 2.055^{\mathrm{b}}$ & $21.332 \pm 0.967^{b}$ & $22.213 \pm 2.209^{b}$ & $24.919 \pm 2.114^{\mathrm{b}}$ \\
\hline & OVX & $24.135 \pm 2.221$ & $16.345 \pm 1.889^{\mathrm{a}}$ & $14.798 \pm 1.929^{\mathrm{a}}$ & $13.025 \pm 2.879^{\mathrm{a}}$ & $11.467 \pm 0.793^{\mathrm{a}}$ \\
\hline & $\mathrm{OVX}+\mathrm{DFO}$ & $24.135 \pm 2.221$ & $25.746 \pm 4.458^{\mathrm{b}}$ & $18.438 \pm 3.256$ & $12.805 \pm 3.333^{\mathrm{a}}$ & $11.247 \pm 1.564^{\mathrm{a}}$ \\
\hline \multirow[t]{3}{*}{ Tb.Th (mm) } & Sham & $0.079 \pm 0.002$ & $0.081 \pm 0.005$ & $0.076 \pm 0.001$ & $0.078 \pm 0.004$ & $0.073 \pm 0.000^{\mathrm{b}}$ \\
\hline & OVX & $0.079 \pm 0.002$ & $0.075 \pm 0.003$ & $0.073 \pm 0.000$ & $0.078 \pm 0.004$ & $0.058 \pm 0.002^{\mathrm{a}}$ \\
\hline & OVX + DFO & $0.079 \pm 0.002$ & $0.081 \pm 0.002$ & $0.078 \pm 0.003^{\mathrm{b}}$ & $0.077 \pm 0.004$ & $0.054 \pm 0.007^{\mathrm{a}}$ \\
\hline \multirow[t]{3}{*}{ Tb.N (/mm) } & Sham & $3.045 \pm 0.210$ & $2.985 \pm 0.096^{\mathrm{b}}$ & $2.815 \pm 0.131$ & $2.781 \pm 0.172^{\mathrm{b}}$ & $2.768 \pm 0.104^{\mathrm{b}}$ \\
\hline & OVX & $3.045 \pm 0.210$ & $2.172 \pm 0.173^{\mathrm{a}}$ & $2.071 \pm 0.248$ & $1.631 \pm 0.154^{\mathrm{a}}$ & $1.646 \pm 0.037^{\mathrm{a}}$ \\
\hline & OVX + DFO & $3.045 \pm 0.211$ & $2.868 \pm 0.203^{\mathrm{b}}$ & $2.355 \pm 0.428$ & $1.643 \pm 0.347^{\mathrm{a}}$ & $1.659 \pm 0.020^{\mathrm{a}}$ \\
\hline \multirow[t]{3}{*}{ Tb.Sp (mm) } & Sham & $0.193 \pm 0.004$ & $0.186 \pm 0.009^{b}$ & $0.206 \pm 0.010$ & $0.219 \pm 0.011^{\mathrm{b}}$ & $0.214 \pm 0.011^{\mathrm{b}}$ \\
\hline & OVX & $0.193 \pm 0.005$ & $0.247 \pm 0.012^{\mathrm{a}}$ & $0.255 \pm 0.017$ & $0.289 \pm 0.026^{\mathrm{a}}$ & $0.283 \pm 0.006^{\mathrm{a}}$ \\
\hline & $\mathrm{OVX}+\mathrm{DFO}$ & $0.193 \pm 0.005$ & $0.202 \pm 0.014^{\mathrm{b}}$ & $0.235 \pm 0.042$ & $0.297 \pm 0.025^{\mathrm{a}}$ & $0.286 \pm 0.005^{\mathrm{a}}$ \\
\hline
\end{tabular}

aSignificant differences compared with sham group; ${ }^{\text {s}}$ significant differences compared with OVX group. BMD, bone mineral density; BV/TV, bone volume fraction; Tb.Th, trabecular thickness; Tb.N, trabecular number; Tb.Sp, trabecular separation.

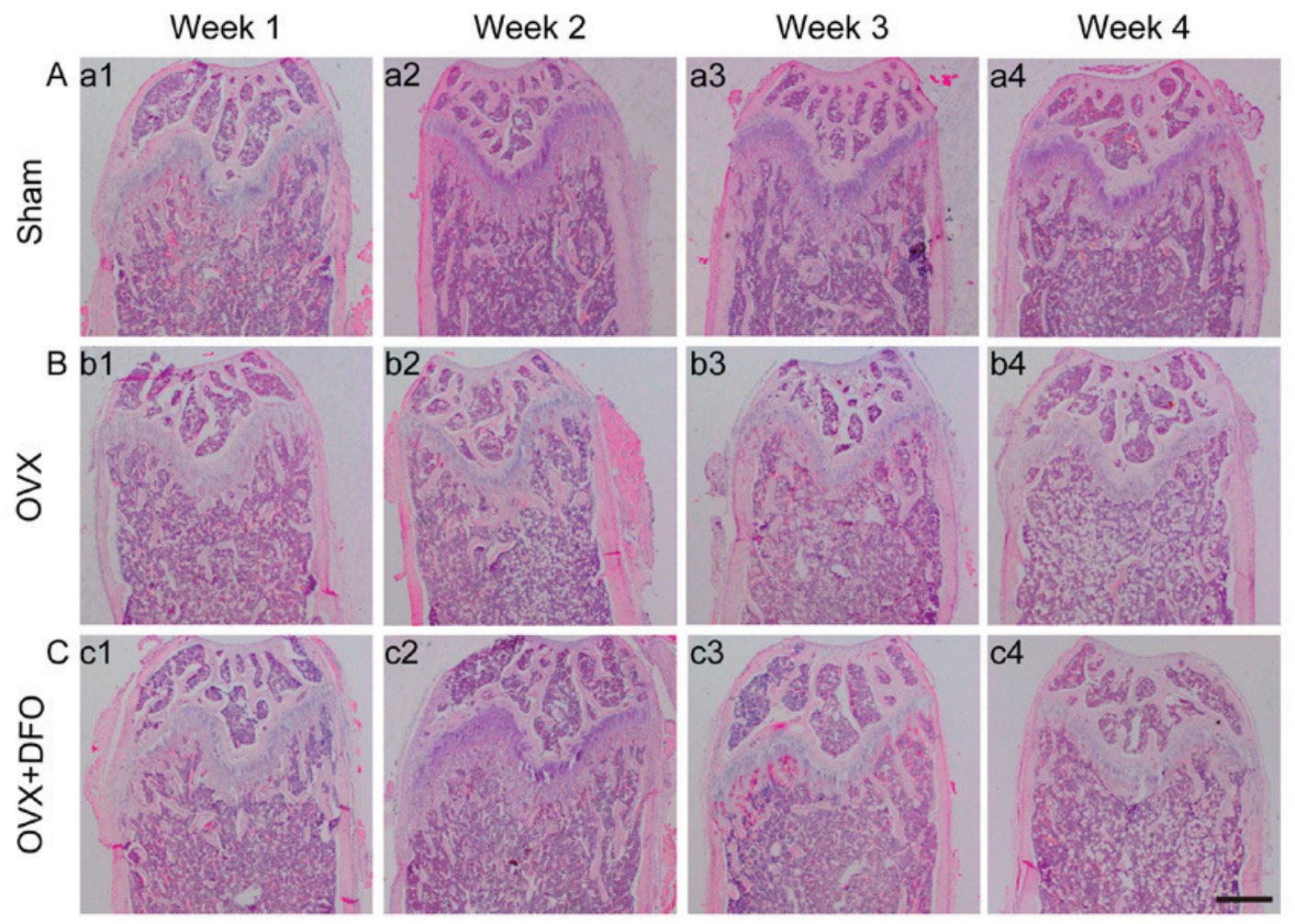

Figure 2. H\&E staining of the distal femur. (A-C) H\&E staining of the distal femur at different points in time in sham group (A-a1-a4), OVX group (B-b1-b4) and OVX + DFO (C-c1-c4) group. Scale bar, $500 \mu \mathrm{m}$. H\&E, hematoxylin and eosin; OVX, ovariectomy; DFO, desferrioxamine.

of blood vessel, the type $\mathrm{H}$ vessel, which plays a pivotal role in combining angiogenesis and osteogenesis (19). Herein, we tried to investigate the vascular changes during our experiment at week 1 and 3. By immunostaining, we observed that the vascular changes in the tibia also showed a similar trend to the bone mass changes (Fig. 4A-L). CD31 ${ }^{+} \mathrm{Emcn}^{+}$type $\mathrm{H}$ blood vessels in OVX mice decreased dramatically at week 1 (Fig. 4B and H), while the OVX + DFO group still maintained a small portion of type $\mathrm{H}$ vessels (Fig. 4C and I). However, type $\mathrm{H}$ vessels were rarely observed in either the OVX or OVX + DFO groups at week 3 after OVX (Fig. 4E, F, $\mathrm{K}$ and $\mathrm{L}$ ), suggesting that $\mathrm{DFO}$ failed to increase the number of 

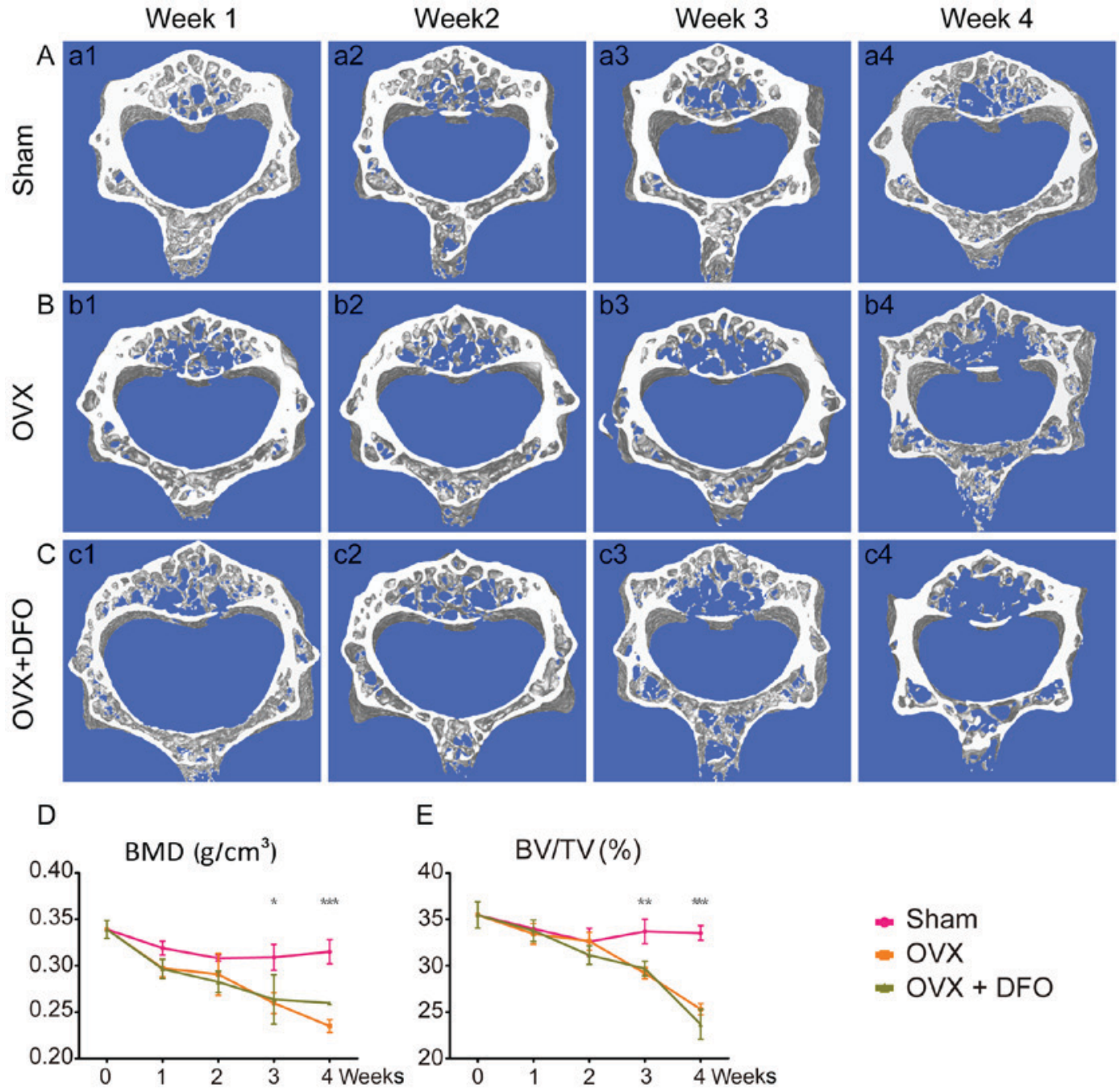

E
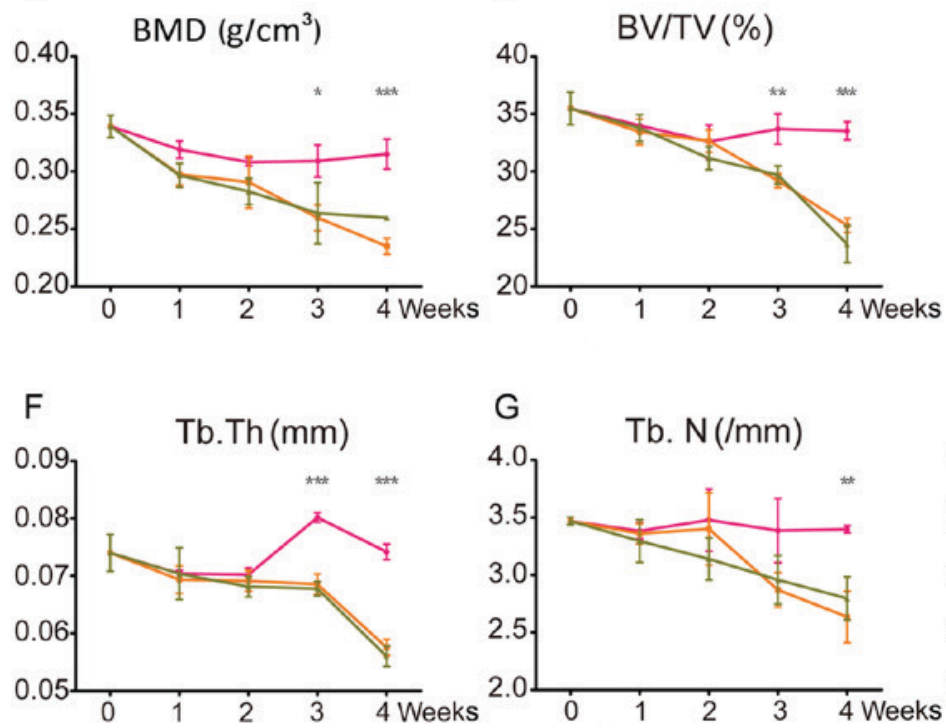

$$
\begin{aligned}
& \text { - Sham } \\
& \text { - OVX } \\
& \text { - OVX + DFO }
\end{aligned}
$$
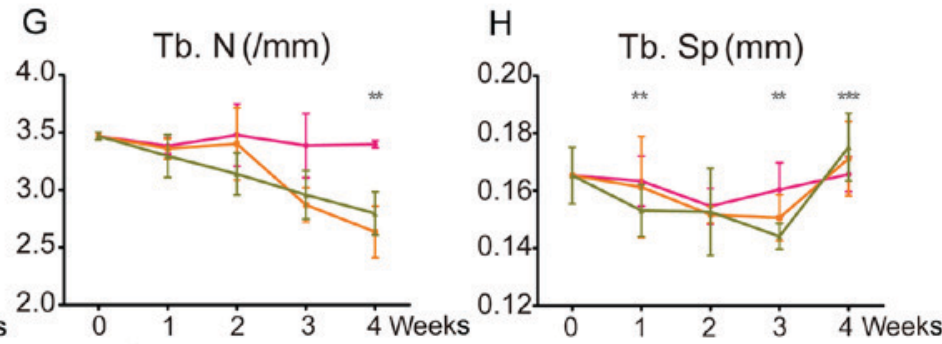

Figure 3. Micro-CT and quantification of bone microarchitecture in the lumbar vertebra. (A-C) 3D micro-CT reconstructed images of the lumbar vertebrae from sham group (A-a1-a4), OVX group (B-b1-b4) and OVX + DFO group (C-c1-c4) at 4 time-points. (D-H) Quantification of BMD (D), BV/TV (E), Tb.Th (F), Tb.N (G) and Tb.Sp (H) from the sham, OVX and OVX + DFO mice. " $\mathrm{P}<0.05 ;{ }^{* *} \mathrm{P}<0.01$; ${ }^{* * *} \mathrm{P}<0.0001$. Micro-CT, micro-computed tomography; OVX, ovariectomy; DFO, desferrioxamine; BMD, bone mineral density; BV/TV, bone volume/tissue volume; Tb.Th, trabecular thickness; Tb.N, trabecular number; Tb.Sp, trabecular separation.

type $H$ vessels at the dose administered. Correlation between vascular and osseous changes implies that blood vessels may be a potential target for osteoporosis therapy.

\section{Discussion}

In this present study, DFO showed a temporary protective effect on OVX-induced osteoporosis, especially in femur. We noticed that the femur showed a greater and sooner response compared to the spine, which is consistent with previous study (22). By immunostaining, we also discovered that type $\mathrm{H}$ vessels and bone mass reduced post-OVX in a time-dependent manner. DFO treatment helped maintain trabecular bone density, restore microarchitecture, and increase the number of type $\mathrm{H}$ vessels in OVX mice at only week 1 and these effects were reversed at weeks 2-4. In addition, our results also revealed that DFO administration had no significant effect on the improvement of spine BMD in OVX mice. One possible cause for this effect is that the concentration of DFO administrated is not able to completely withstand the bone loss caused by continual estrogen loss. It will be our future direction to make in-depth investigation in order to study the dynamics 
Table II. BMD and trabecular microarchitecture of lumbar at different time-points.

\begin{tabular}{llrrrrr}
\hline Parameters & \multicolumn{1}{c}{ Group } & Week 0 & Week 1 & Week 2 & Week 3 & Week 4 \\
\hline BMD $\left(\mathrm{g} / \mathrm{cm}^{3}\right)$ & Sham & $0.339 \pm 0.010$ & $0.319 \pm 0.007$ & $0.308 \pm 0.003$ & $0.309 \pm 0.014^{\mathrm{b}}$ & $0.315 \pm 0.013^{\mathrm{b}}$ \\
& OVX & $0.339 \pm 0.010$ & $0.297 \pm 0.009$ & $0.291 \pm 0.023$ & $0.260 \pm 0.011^{\mathrm{a}}$ & $0.235 \pm 0.007^{\mathrm{a}}$ \\
& OVX + DFO & $0.339 \pm 0.010$ & $0.297 \pm 0.010$ & $0.283 \pm 0.011$ & $0.264 \pm 0.027^{\mathrm{a}}$ & $0.260 \pm 0.002^{\mathrm{a}, \mathrm{b}}$ \\
BV/TV (\%) & Sham & $35.472 \pm 1.409$ & $33.994 \pm 0.217$ & $32.583 \pm 1.452$ & $33.693 \pm 1.314^{\mathrm{b}}$ & $33.526 \pm 0.788^{\mathrm{b}}$ \\
& OVX & $35.472 \pm 1.409$ & $33.431 \pm 1.126$ & $32.642 \pm 0.972$ & $29.180 \pm 0.603^{\mathrm{a}}$ & $25.301 \pm 0.610^{\mathrm{a}}$ \\
& OVX + DFO & $35.472 \pm 1.409$ & $33.775 \pm 1.163$ & $31.146 \pm 1.002$ & $29.696 \pm 0.772^{\mathrm{a}}$ & $23.684 \pm 1.609^{\mathrm{a}}$ \\
Tb.Th $(\mathrm{mm})$ & Sham & $0.074 \pm 0.003$ & $0.071 \pm 0.000$ & $0.070 \pm 0.001$ & $0.080 \pm 0.000^{\mathrm{b}}$ & $0.074 \pm 0.001^{\mathrm{b}}$ \\
& OVX & $0.074 \pm 0.003$ & $0.069 \pm 0.002$ & $0.069 \pm 0.002$ & $0.069 \pm 0.002^{\mathrm{a}}$ & $0.058 \pm 0.001^{\mathrm{a}}$ \\
& OVX + DFO & $0.074 \pm 0.003$ & $0.070 \pm 0.004$ & $0.068 \pm 0.002$ & $0.068 \pm 0.001^{*}$ & $0.056 \pm 0.002^{*}$ \\
Tb.N $(/ \mathrm{mm})$ & Sham & $3.469 \pm 0.033$ & $3.384 \pm 0.069$ & $3.479 \pm 0.270$ & $3.387 \pm 0.278$ & $3.398 \pm 0.032^{\mathrm{b}}$ \\
& OVX & $3.469 \pm 0.033$ & $3.360 \pm 0.087$ & $3.401 \pm 0.314$ & $2.871 \pm 0.150$ & $2.636 \pm 0.224^{\mathrm{a}}$ \\
& OVX + DFO & $3.469 \pm 0.033$ & $3.295 \pm 0.184$ & $3.140 \pm 0.183$ & $2.957 \pm 0.211$ & $2.797 \pm 0.189^{\mathrm{a}}$ \\
Tb.Sp $(\mathrm{mm})$ & Sham & $0.165 \pm 0.009$ & $0.163 \pm 0.008^{\mathrm{b}}$ & $0.155 \pm 0.006$ & $0.160 \pm 0.009^{\mathrm{b}}$ & $0.166 \pm 0.006^{\mathrm{b}}$ \\
& OVX & $0.165 \pm 0.010$ & $0.161 \pm 0.018^{\mathrm{a}}$ & $0.152 \pm 0.003$ & $0.151 \pm 0.008^{\mathrm{a}}$ & $0.171 \pm 0.013^{\mathrm{a}}$ \\
& OVX + DFO & $0.165 \pm 0.010$ & $0.153 \pm 0.009^{\mathrm{b}}$ & $0.153 \pm 0.015$ & $0.144 \pm 0.004^{\mathrm{a}}$ & $0.175 \pm 0.012^{\mathrm{a}}$ \\
\hline
\end{tabular}

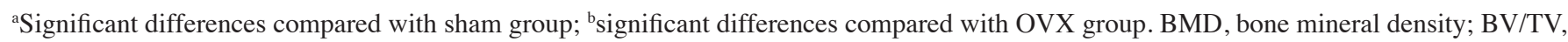
bone volume fraction; Tb.Th, trabecular thickness; Tb.N, trabecular number; Tb.Sp, trabecular separation.

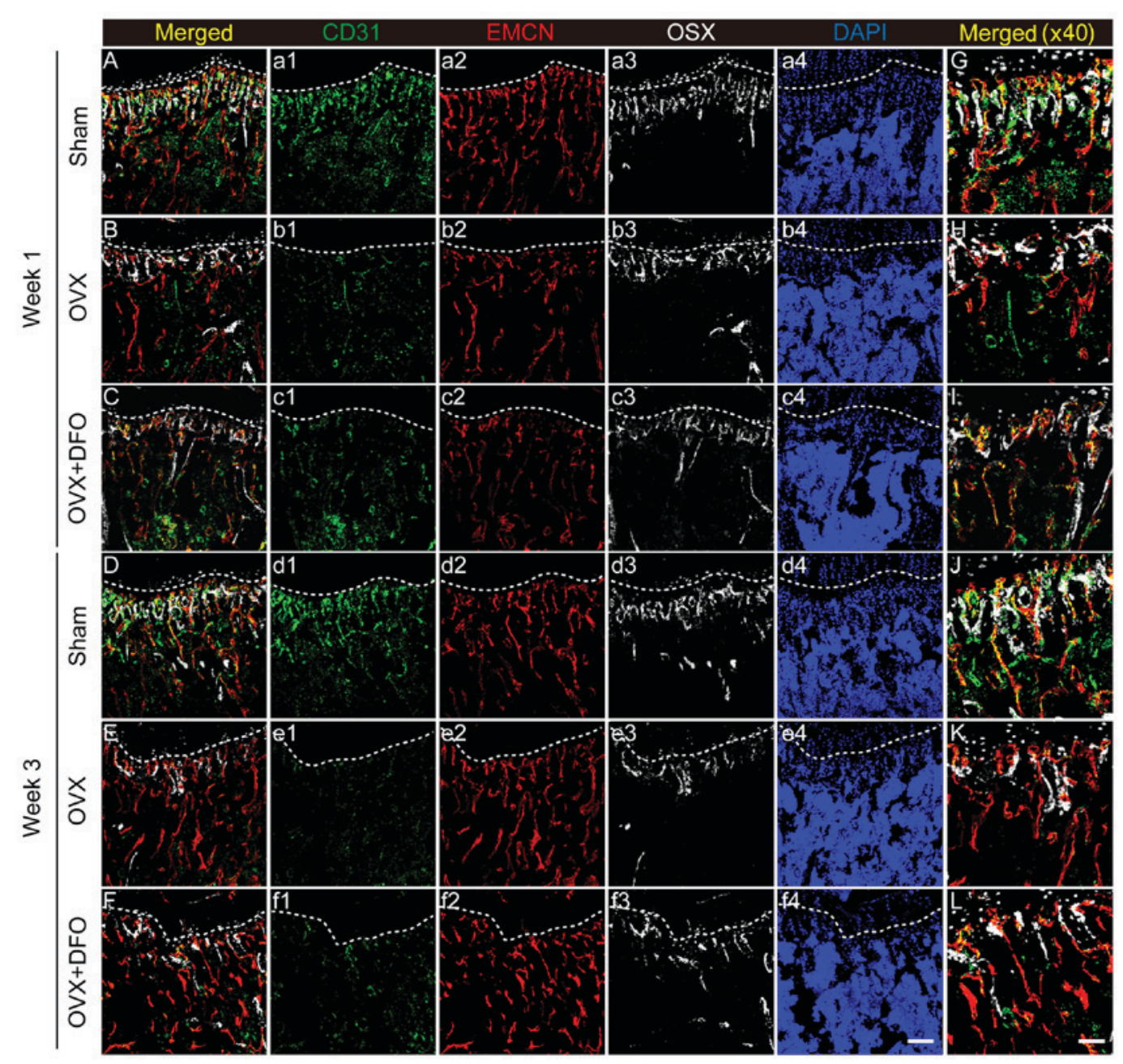

Figure 4. Changes of type H vessels in osteoporotic mice. (A-F) Tibia immunostaining (x20) for CD31 (green), endomucin (red), osterix (gray) and DAPI (blue) of the sham (A and D), OVX (B and E) and OVX + DFO (C and F) group mice at week 1 (A-C) and week 3 (D-F), respectively. (G-L) Higher magnification (x40) view of metaphysis region. The line indicates the boundary of the metaphysis and growth plate. OVX, ovariectomy; DFO, desferrioxamine. Scale bar, $100 \mu \mathrm{m}$ in $\mathrm{F}$ (f4); $200 \mu \mathrm{m}$ in $\mathrm{L}$. 
of osteogenesis and angiogenesis using DFO gradients and to determine the underlying mechanism.

DFO has been shown to increase angiogenesis via the HIF pathway in bone (23). DFO therapy can optimize bone regeneration in mandibular distraction osteogenesis by increasing the number of osteocytes and vessels $(24,25)$. DFO released from poly lactic-co-glycolic acid promotes healing of osteoporotic bone defects by enhancing the differentiation of MSCs and endothelial tubule formation (26). These results show that DFO can accelerate fracture healing and bone regeneration by inducing new blood vessel formation. In our study, DFO increased bone mass and enhanced type $H$ vessels at week 1 in the femur, which may be attributed to HIF pathways. However, at week 3, DFO failed to increase the number of type $H$ vessels and restore BMD. One cause may be that OVX-induced estrogen loss overwhelms the osteogenic and angiogenic effect of DFO.

Accumulating evidence suggests that local blood supply or decreased angiogenesis play pivotal roles in osteoporosis (27-29). Osteoporotic fracture is one of the most frequent complications of bone loss, easily leading to disability and death in the elderly. Healing of fragility fractures would be prolonged with the decreased levels of angiogenesis and MSCs caused by OVX (30). Low-magnitude high-frequency vibration treatment could enhance fracture healing by increasing blood flow and angiogenesis in the OVX mouse model (31). This has great clinical significance in the improvement of osteoporosis and prevention of fragility fractures by promoting blood vessel formation in bone. Type $\mathrm{H}$ vessels combine angiogenesis and osteogenesis in murine bone, which decreases along with ageing (19,32,33). DFO could increase type $\mathrm{H}$ vessel formation and improve BMD in aged mice (19). When looked separately, $\mathrm{CD} 31^{+}$ECs showed more significant change than $\mathrm{EMCN}^{+} \mathrm{ECs}$, resulting in dramatic change of type $\mathrm{H}$ vessels. The vessel changes we observed in OVX mice models are quite similar. $\mathrm{EMCN}^{+}$ECs appeared to be comparable at week-3 after OVX while $\mathrm{CD} 31^{+}$ECs decreased dramatically. As a result, type $\mathrm{H}$ vessel was significantly reduced in OVX mice and DFO treatment attenuated the effect temporarily. This would be due to type $\mathrm{H}$ vessels (CD31 expression) are more sensitive to physiological and/or pathological stress and would be considered as an early marker for bone status. Studies need to be pursued to investigate the relationship between type $\mathrm{H}$ vessels and bone formation/maintenance, especially in pathological situations.

As an angiogenic agent, DFO has potential to active certain vascular pathways. How these two molecular effects coordinate to achieve a protective effect against osteoporotic process is still unknown. Our study offers preliminary information for the clinical therapeutic treatment of osteoporosis. Coupling different pathways, e.g., vascular and osseous, may achieve a better therapeutic effect. Scientists and clinicians need to further test specific therapeutic options, e.g., DFO, to fully understand their functions.

\section{Acknowledgements}

We thank all the staffs of the animal facility for maintaining our experimental animals. This study was supported by the National Natural Science Foundation of China (grant nos. 81273090 and 81572179), the Clinical Special Program of Jiangsu Province (grant no. BL2014044), Clinical Key
Diseases' Diagnosis and Treatment of Suzhou (grant no. LCZX201305), Science and Technology Project of Suzhou (grant no. SYS201637).

\section{References}

1. Kanis JA: Assessment of fracture risk and its application to screening for postmenopausal osteoporosis: Synopsis of a WHO report. WHO Study Group. Osteoporos Int 4: 368-381, 1994.

2. Kanis JA, McCloskey EV, Johansson H and Oden A: Approaches to the targeting of treatment for osteoporosis. Nat Rev Rheumatol 5: 425-431, 2009.

3. Harvey N, Dennison E and Cooper C: Osteoporosis: Impact on health and economics. Nat Rev Rheumatol 6: 99-105, 2010.

4. Rachner TD, Khosla S and Hofbauer LC: Osteoporosis: Now and the future. Lancet 377: 1276-1287, 2011

5. Garnero P: New developments in biological markers of bone metabolism in osteoporosis. Bone 66: 46-55, 2014.

6. Harvey N, Dennison E and Cooper C: Osteoporosis: A lifecourse approach. J Bone Miner Res 29: 1917-1925, 2014.

7. Hendrickx G, Boudin E and Van Hul W: A look behind the scenes: The risk and pathogenesis of primary osteoporosis. Nat Rev Rheumatol 11: 462-474, 2015.

8. Reid IR: Short-term and long-term effects of osteoporosis therapies. Nat Rev Endocrinol 11: 418-428, 2015.

9. Chang J, Wang Z, Tang E, Fan Z, McCauley L, Franceschi R, Guan K, Krebsbach PH and Wang CY: Inhibition of osteoblastic bone formation by nuclear factor-kappaB. Nat Med 15: 682-689, 2009.

10. Lewiecki EM: New targets for intervention in the treatment of postmenopausal osteoporosis. Nat Rev Rheumatol 7: 631-638, 2011.

11. Maeda K, Kobayashi Y, Udagawa N, Uehara S, Ishihara A, Mizoguchi T, Kikuchi Y, Takada I, Kato S, Kani S, et al: Wnt5a-Ror2 signaling between osteoblast-lineage cells and osteoclast precursors enhances osteoclastogenesis. Nat Med 18: 405-412, 2012.

12. Scholtysek C, Katzenbeisser J, Fu H, Uderhardt S, Ipseiz N, Stoll C, Zaiss MM, Stock M, Donhauser L, Böhm C, et al: PPAR $\beta / \delta$ governs Wnt signaling and bone turnover. Nat Med 19: 608-613, 2013.

13. Tomlinson RE and Silva MJ: Skeletal blood flow in bone repair and maintenance. Bone Res 1: 311-322, 2013.

14. Colnot C: Cellular and molecular interactions regulating skeletogenesis. J Cell Biochem 95: 688-697, 2005.

15. Brandi ML and Collin-Osdoby P: Vascular biology and the skeleton. J Bone Miner Res 21: 183-192, 2006.

16. Wan C, Gilbert SR, Wang Y, Cao X, Shen X, Ramaswamy G, Jacobsen KA, Alaql ZS, Eberhardt AW, Gerstenfeld LC, et al: Activation of the hypoxia-inducible factor-1alpha pathway accelerates bone regeneration. Proc Natl Acad Sci USA 105: 686-691, 2008.

17. Schipani E, Maes C, Carmeliet G and Semenza GL: Regulation of osteogenesis-angiogenesis coupling by HIFs and VEGF. J Bone Miner Res 24: 1347-1353, 2009.

18. Dirckx N, Van Hul M and Maes C: Osteoblast recruitment to sites of bone formation in skeletal development, homeostasis and regeneration. Birth Defects Res C Embryo Today 99: 170-191, 2013.

19. Kusumbe AP, Ramasamy SK and Adams RH: Coupling of angiogenesis and osteogenesis by a specific vessel subtype in bone. Nature 507: 323-328, 2014.

20. Qu ZH, Zhang XL, Tang TT and Dai KR: Promotion of osteogenesis through beta-catenin signaling by desferrioxamine. Biochem Biophys Res Commun 370: 332-337, 2008.

21. Donneys A, Weiss DM, Deshpande SS, Ahsan S, Tchanque-Fossuo CN, Sarhaddi D, Levi B, Goldstein SA and Buchman SR: Localized deferoxamine injection augments vascularity and improves bony union in pathologic fracture healing after radiotherapy. Bone 52: 318-325, 2013.

22. Liu XL, Li CL, Lu WW, Cai WX and Zheng LW: Skeletal site-specific response to ovariectomy in a rat model: Change in bone density and microarchitecture. Clin Oral Implants Res 26: 392-398, 2015.

23. Drager J, Harvey EJ and Barralet J: Hypoxia signalling manipulation for bone regeneration. Expert Rev Mol Med 17: e6, 2015. 
24. Farberg AS, Sarhaddi D, Donneys A, Deshpande SS and Buchman SR: Deferoxamine enhances bone regeneration in mandibular distraction osteogenesis. Plast Reconstr Surg 133: 666-671, 2014.

25. Donneys A, Farberg AS, Tchanque-Fossuo CN, Deshpande SS and Buchman SR: Deferoxamine enhances the vascular response of bone regeneration in mandibular distraction osteogenesis. Plast Reconstr Surg 129: 850-856, 2012.

26. Jia P, Chen H, Kang H, Qi J, Zhao P, Jiang M, Guo L, Zhou Q, Qian ND, Zhou HB, et al: Deferoxamine released from poly(lactic-co-glycolic acid) promotes healing of osteoporotic bone defect via enhanced angiogenesis and osteogenesis. J Biomed Mater Res A 104: 2515-2527, 2016

27. Senel K, Baykal T, Seferoglu B, Altas EU, Baygutalp F, Ugur M and Kiziltunc A: Circulating vascular endothelial growth factor concentrations in patients with postmenopausal osteoporosis Arch Med Sci 9: 709-712, 2013.

28. Kanczler JM and Oreffo RO: Osteogenesis and angiogenesis: The potential for engineering bone. Eur Cell Mater 15: 100-114, 2008.
29. Griffith JF, Wang YX, Zhou H, Kwong WH, Wong WT, Sun YL, Huang Y, Yeung DK, Qin L and Ahuja AT: Reduced bone perfusion in osteoporosis: Likely causes in an ovariectomy rat model. Radiology 254: 739-746, 2010.

30. Cheung WH, Miclau T, Chow SK, Yang FF and Alt V: Fracture healing in osteoporotic bone. Injury 47 (Suppl 2): S21-S26, 2016.

31. Cheung WH, Sun MH, Zheng YP, Chu WC, Leung AH, Qin L, Wei FY and Leung KS: Stimulated angiogenesis for fracture healing augmented by low-magnitude, high-frequency vibration in a rat model-evaluation of pulsed-wave doppler, 3-D power Doppler ultrasonography and micro-CT microangiography. Ultrasound Med Biol 38: 2120-2129, 2012.

32. le Noble F and le Noble J: Bone biology: Vessels of rejuvenation. Nature 507: 313-314, 2014.

33. Kusumbe AP and Adams RH: Osteoclast progenitors promote bone vascularization and osteogenesis. Nat Med 20: 1238-1240, 2014. 